\title{
INTERMUNICIPALIDAD COMO UN ARREGLO INSTITUCIONAL EMERGENTE: EL CASO DEL SUMINISTRO DE AGUA EN LA ZONA METROPOLITANA DE AGUASCALIENTES, MÉXICO*
}

\author{
Raúl Pacheco-Vega \\ Centro de Investigación y Docencia Económicas, México \\ raul.pacheco-vega@cide.edu
}

\begin{abstract}
RESUMEN
La gestión del servicio público del agua es un proceso complejo que requiere de la implementación de una serie de mecanismos de rendición de cuentas, coordinación y regulación para garantizar el suministro continuo del vital líquido al mayor número de personas, maximizando la cobertura tanto horaria como espacial. El artículo examina el caso de una urbe mexicana que se ha ido expandiendo hasta conformar una zona metropolitana, Aguascalientes, analizando de manera crítica el argumento que plantea que el manejo intermunicipal de servicios públicos emerge como una respuesta a las necesidades crecientemente complejas de las regiones metropolitanas de ofrecer un servicio de calidad a una población cada vez más exigente y creciente.
\end{abstract}

Palabras clave: Intermunicipalidad, Gobernanza del agua, Instituciones, México.

* Agradezco a los dictaminadores anónimos de la Revista de Gestión Pública por sus sugerencias sobre la situación del artículo en el contexto global de la literatura, los métodos de recolección de datos, y las dimensiones foco de investigación del trabajo. 


\title{
INTERMUNICIPALITY AS AN EMERGENT INSTITUTIONAL ARRANGEMENT: \\ THE WATER SUPPLY CASE IN THE METROPOLITAN AREA OF AGUASCALIENTES, MEXICO
}

\begin{abstract}
Public water supply management is a complex process that requires the implementation of a series of accountability, coordination and regulatory mechanisms in order to guarantee continual supply of this vital liquid to the maximum number of people, maximizing spatial and hourly coverage. This article examines the case of a Mexican city that has been expanding until reaching the size of a metropolitan zone, i.e. Aguascalientes, analyzing in a critical manner the argument that intermunicipal governance of public services emerges as a response to increasingly complex needs of metropolitan regions, which need to offer quality services to a growing and demanding population.
\end{abstract}

Keywords: Intermunicipality, Water governance, Institutions, México. 


\section{INTRODUCCIÓN}

La gestión del servicio público del agua es un proceso complejo que requiere de la implementación de una serie de mecanismos de rendición de cuentas, coordinación y regulación para garantizar el suministro continuo del vital líquido al mayor número de personas, maximizando la cobertura tanto horaria (número de horas que se está conectado a la red pública de provisión de agua) como espacial (dimensiones espaciales del área geográfica de suministro del líquido). La continua expansión urbana y el incremento en la presión que resienten los sistemas de suministro de agua (también llamados organismos operadores) dado el crecimiento poblacional tanto por migración interna como por explosión demográfica han dado lugar a la necesidad de ver los problemas de provisión del servicio público de agua potable, alcantarillado y saneamiento no solamente como un problema de una urbe sino una problemática intermunicipal.

En este artículo, examino el caso de una urbe mexicana que se ha ido expandiendo hasta conformar una zona metropolitana, Aguascalientes. Dicha ciudad ha tenido problemas de suministro de agua desde mediados de los años 1980, en particular debido a la ineficiencia del organismo operador de agua de garantizar el suministro continuo de agua potable, servicios de tratamiento de efluentes residuales e infraestructura de alcantarillado adecuada. La problemática de los servicios públicos municipales de agua no ha afectado solamente a la creciente y expandida urbe de Aguascalientes, sino también a sus vecinos directos. Dada la urbanización explosiva de Aguascalientes, y que las dos municipalidades colindantes (Jesús María y San Francisco de los Romo) han comenzado también a ser ciudades de mayor área espacial y relevancia en la actividad socioeconómica del estado de Aguascalientes, la creación de una zona metropolitana de Aguascalientes y un modelo de gobernanza metropolitana de tipo intermunicipal parece una conclusión directa y una opción de política relativamente sencilla de implementar. Sin embargo, la evidencia empírica arroja una intermunicipalidad emergente y no consolidada.

El objetivo del artículo es analizar el manejo de agua en la zona metropolitana de Aguascalientes a través del lente analítico de los procesos cooperativos de suministro de servicios públicos, con el fin de contribuir a nuestro conocimiento sobre los mecanismos de emergencia de instituciones de cooperación. La metodología utilizada sigue el modelo de caso de estudio (Dooley 2002, Flyvbjerg 2011, Gilson 2012, Seawright y Gerring 2008). A fin de estudiar la emergencia incipiente de una institución de intermunicipalidad en dicha zona metropolitana, durante el periodo de septiembre del 2012 a septiembre del 2014 realicé entrevistas a profundidad a actores clave, visitas de campo a las tres diferentes municipalidades que 
conforman la zona metropolitana de Aguascalientes, recolecté información de fuentes secundarias tanto digitales como físicas del Instituto Nacional de Geografía, Estadística e Informática de México (INEGI), y de los gobiernos municipales de cada una de las ciudades conurbadas en Aguascalientes, así como del gobierno estatal.

En este artículo examino el concepto de intermunicipalidad, analizando de manera crítica el argumento en la literatura académica sobre el tema en que se indica que el manejo intermunicipal de servicios públicos emerge como una respuesta a las necesidades crecientemente complejas de las regiones metropolitanas de ofrecer un servicio de calidad a una población cada vez más exigente y creciente. En este sentido, mi investigación contribuye a la literatura sobre modelos cooperativos de gobernanza urbana mediante el exámen riguroso de la evidencia disponible en el caso de estudio bajo análisis y la aplicación de los conceptos conocidos en la literatura sobre las dimensiones de cooperación y la formulación y emergencia de instituciones cooperativas. Los casos empíricos de estudio de la emergencia de la intermunicipalidad en México son pocos si bien en el ámbito teórico el trabajo de Rodríguez-Oreggia y de Ramírez de la Cruz son importantes (Ramírez de la Cruz 2012, Rodríguez-Oreggia y Tuirán Gutiérrez 2006), y en los casos de estudio conocidos de manera empírica está el trabajo de José Santos Zavala en San Luis Potosí, San Luis Potosí (Alcalde Alderete 2003, Santos Zavala 2004) y de Lourdes Amaya Ventura sobre Pachuca, Hidalgo (Amaya Ventura 2011). Este trabajo por ende contribuye tanto de manera teórica como empírica mediante el análisis del caso de la zona metropolitana de Aguascalientes. El artículo se desarrolla de la siguiente forma: en la segunda sección, posterior a ésta inicial, describo las teorías de la intermunicipalidad y realizo una revisión exhaustiva de la literatura tanto de fuentes anglosajonas como hispanoparlantes. Si bien el caso de estudio está localizado en México, las lecciones aprendidas del mismo pueden ser fácilmente transferidas a otros países.

En la tercera sección establezco algunas hipótesis sobre cómo contribuiría un arreglo intermunicipal a la gobernanza del agua en una zona metropolitana. En la cuarta describo el caso de estudio de la zona metropolitana de Aguascalientes y sus tres municipios conurbados: Jesús María, Aguascalientes y San Francisco de los Romo, al mismo tiempo que aplico el recorte analítico de intermunicipalidad al mismo, extrayendo las enseñanzas que nos ofrece las peculiaridades del caso de la zona metropolitana de Aguascalientes. En la quinta sección, cierro con una serie de postulados y preguntas sobre cómo podríamos implementar la intermunicipalidad de una forma más robusta. Es importante hacer notar que la evidencia empírica que se deriva de los tres casos de estudio 
muestra que la intermunicipalidad en Aguascalientes es apenas incipiente. El artículo demuestra que para poder implementar un arreglo institucional intermunicipal se requiere un grado de coordinación extraordinario, y un modelo de reglas formales e informales de organización robusto.

\section{¿QUÉ SABEMOS SOBRE LA INTERMUNICIPALIDAD? UNA REVISIÓN DE LA LITERATURA}

En general, los servicios públicos son competencia de la administración pública municipal, exceptuando en casos tales como Uruguay donde la gestión del manejo de agua fue durante mucho tiempo responsabilidad de la federación, o del gobierno nacional. La gestión pública del suministro de agua es responsabilidad, al menos en México, directamente del municipio como resultado de la reforma a la Constitución Política de los Estados Unidos Mexicanos en su artículo 115 constitucional (Pacheco-Vega 2009, 2014a). La reforma se inició en 1983 dando un carácter más relevante al municipio en la estructura organizacional y en la arquitectura institucional al hacer a los gobiernos locales responsables de los servicios públicos. En 1999, México logró la reforma que adicionó los servicios de suministro de agua potable, alcantarillado y saneamiento a la lista de servicios públicos que eran responsabilidad municipal (Fernández Ruiz 2001, López 2000).

¿Qué es un arreglo institucional intermunicipal? En mi visión, el concepto de intermunicipalidad tiene cuando menos dos dimensiones fundamentales: la primera es la dimensión espacial y geográfica, donde un arreglo intermunicipal surge como resultado de la expansión urbana y de la transformación de las urbes por el crecimiento poblacional, la inmigración y el incremento en las poblaciones flotantes en ciudades limítrofes o con probabilidades de colindancia en periodos relativamente cortos. La segunda dimensión es la colaborativa e institucional, donde el arreglo intermunicipal deriva del establecimiento de una serie de reglas formales e informales de cooperación entre los diferentes municipios o ciudades. Es de hacer notar que por definición, la intermunicipalidad establece que debe existir cooperación entre los municipios (Pacheco-Vega 2013a, Ramírez de la Cruz 2012). Es decir, las reglas formales e informales son reglas cooperativas, no confrontacionales. En este sentido, el arreglo institucional intermunicipal es un arreglo potencialmente virtuoso. También es importante hacer notar que así como puede haber diversidad institucional, puede haber erosión institucional (Ostrom 2005).

Los arreglos institucionales intermunicipales están asociados íntimamente con la definición de gobernanza, por un lado, y de gobernanza metropolitana, por el otro (Feiock 2004, Feiock 2008). Uno no puede conceptualizar una relación colaborativa entre ciudades sin tomar en 
cuenta que en el proceso de establecimiento de dichas colaboraciones, cada gobierno local tendrá que ceder cierto grado de control, y establecer una serie de reglas formales e informales que establezcan de manera explícita los mecanismos de interacción entre los agentes gubernamentales, los políticos, los burócratas y el personal de cada agencia que tenga por objetivo una relación de colaboración con sus contrapartes en otros municipios y/o ciudades. Al hacer este tipo de cesión de control, los gobiernos locales se ven entonces forzados a analizar modelos de gobierno que son menos jerárquicos y de arriba hacia abajo, y considerar modelos que empoderan tanto a los ciudadanos como a los mismos burócratas. Esto obliga forzosamente a establecer o implementar un modelo ya no de gobierno sino de gobernanza (Digaetano y Lawless 1999, Hughes 2012, Pacheco-Vega y Vega 2008).

De acuerdo con Lefevre (1998), existen dos modalidades de arreglos institucionales en la gobernanza metropolitana: los supramunicipales y los intermunicipales. La supramunicipalidad constituye un modelo de gobernanza urbana de tipo jerárquico con múltiples poderes y con legitimidad política directa. La intermunicipalidad, por el contrario, tiene diversos elementos que son más maleables y fluidos. Por ejemplo, para Lefevre, las estructuras intermunicipales raramente tienen autonomía financiera. Sin embargo, para una visión contrapuesta, puede verse el trabajo de Warner, Bel y Mur, quienes demuestran que la estructura de cooperación intermunicipal en la gestión pública del servicio de recolección y disposición de basura en España en las mancomunidades y las comarcas tiene un grado mayor de independencia financiera que si cada municipio tuviera que hacerse responsable de la colección, tratamiento y disposición final de sus residuos sólidos municipales (Germà Bel y Mur 2009, Warner y Bel 2008). Independientemente de los grados de autonomía, la ventaja de los arreglos intermunicipales por encima de la supramunicipalidad es que hay un grado substancial de legitimidad que proviene del establecimiento de relaciones cooperativas entre los diferentes niveles de gobierno y entre los mismos gobiernos locales.

El surgimiento de los arreglos intermunicipales de cooperación inicia en parte como resultado del rescalamiento (Cohen y McCarthy 2014, Nelles y Durand 2012) de las acciones gubernamentales de provisión de servicios públicos. Por ejemplo, en el caso de los Estados Unidos de Norteamérica, este tipo de acciones cooperativas inicia en los condados, en las comarcas en españa, y en las comunidades de comunes (communautés de communes) en Francia (Germà Bel y Warner 2014). De acuerdo con Bel y Warner, la cooperación es una forma de gobernanza por redes, si bien se estoss autores se manifiestan escépticos de la capacidad de los arreglos horizontales de 
colaboración, sobre todo porque asumen que al transformarse los nodos de control del poder de tener una organización jerárquica a transformarse en una red de política pública (Huppé, Creech, y Knoblauch 2012, Moynihan 2009), también se cambia el tipo de instrumento de política pública, de ser un instrumento regulatorio como el comando y control o los estándares con sanciones asociadas, a un instrumento de persuasión y/o suministro de información (Pacheco-Vega y Nemetz 2001, Vedung 1998). La suposición de Bel y Warner no es del todo correcta ya que la gobernanza por redes no necesariamente implica o presupone una transformación en la caja de herramientas de política pública utilizada por los gobiernos tanto municipales, como por el arreglo intermunicipal colaborativo.

Los arreglos intermunicipales también emergen generalmente como resultado de la necesidad de fortalecer una posición política originalmente débil. Los municipios, dada su precaria posición desde la perspectiva de la construcción de políticas públicas y la gestión pública, han comenzado a buscar modelos innovadores de gestión en los que se vean reducidos costos, se maximicen eficiencias y se minimicen redundancias. Es interesante ver que la reacción por parte de los municipios es de tipo colaborativa, sobre todo en circunstancias como las que hemos visto en años recientes, donde el diseño y establecimiento de agendas públicas ocurre en las escalas federales o supranacionales, mientras que la implementación de políticas públicas ocurre en las escalas locales y municipales. Por ello, como indican Hurst y Van Montfort:

[...] independientemente de la escala, ámbito, autonomía y posición en el sistema nacional de gestión pública, una serie de desarrollos en los últimos 50 años ha puesto presión en los gobiernos locales para que tengan un buen desempeño, dominio e incluso han cuestionado su existencia (Hulst y Van Montfort 2007b: 3)

Es de hacer notar que un arreglo intermunicipal no necesariamente requiere de la consolidación de las municipalidades (Germà Bel y Warner 2014, Hulst y Van Montfort 2007b), como lo argumentan Bel y Warner en su estudio sobre cooperación intermunicipal y costos. Por el contrario, la intermunicipalidad tiene como ventaja la flexibilidad, maleabilidad y fluidez que le da el ser un arreglo institucional cooperativo. Por ejemplo, en la gestión de la política climática de adaptación, que es un problema fundamentalmente local, la cooperación intermunicipal permite fortalecer las capacidades institucionales, técnicas y de capital humano de las ciudades que entran en un arreglo intermunicipal, como en el caso de algunas municipalides en Sudáfrica (Pasquini y Shearing 2014). 
Un caso mexicano interesante de arreglos intermunicipales colaborativos es el del caso de la cuenca del Río Ayuquila (Montero et al. 2006), donde diez municipalidades dentro de la cuenca del Río Ayuquila formaron una asociación colaborativa para reducir la contaminación del río, si bien sus objetivos macro eran trabajar de forma conjunta para mejorar las condiciones de vida y promover el desarrollo sustentable en sus límites administrativos. Es importante hacer notar que en el caso de la asociación intermunicipal de la cuenca del Río Ayuquila, los participantes se enfocaron en establecer mecanismos para garantizar que su arreglo institucional colaborativo fuera sostenible en el mediano y largo plazo, incluyendo la búsqueda de apoyos de políticos en la escala gubernamental municipal, fortaleciendo sus capacidades técnicas y garantizando la continuidad del mismo (Montero et al. 2006).

No todos los casos de cooperación intermunicipal han tenido éxito, en particular porque no siempre existen este tipo de iniciativas enfocadas a la sostenibilidad y robustecimiento de las relaciones colaborativas interinstitucionales entre los municipios (Pacheco-Vega 2013b). Por ejemplo, en el caso de la cuenca Lerma-Chapala, en el centro de México, la colaboración intermunicipal e interestatal en materia de aguas residuales ha sido bastante poco efectiva, a pesar de existir un consejo de cuenca longevo (fundado en 1993), y que se ha invertido mucho tiempo y dinero en establecer las relaciones de colaboración entre los diferentes niveles de gobierno (Pacheco-Vega y Basurto 2008, Pacheco-Vega 2007a). Esto es problemático ya que los consejos de cuenca, así como los comités de aguas subterráneas son innovaciones institucionales diseñadas específicamente para fortalecer la colaboración entre las diferentes instancias, los usuarios, y otros actores incluyendo la academia, la industria y los servicios públicos municipales (CONAGUA 2010, Griffin 1999, Pacheco-Vega 2011). Sin embargo, una revisión detallada de la literatura nos permite ver que hay muchos casos en los cuales la intermunicipalidad resulta benéfica y con efectos positivos, en comparación, por ejemplo, con un arreglo institucional jerárquico o desintegrado.

Los arreglos de cooperación intermunicipal pueden ser una de las posibles soluciones para reducir costos de suministro de servicios públicos, como en el caso de la gestión de residuos sólidos urbanos, precisamente evitando la privatización (Germà Bel y Mur 2009, Germà Bel y Warner 2008). El trabajo de Bel y Warner hace una evaluación de los costos de la implementación de arreglos institucionales intermunicipales para la provisión de servicios públicos (Germà Bel y Warner 2014). Dado que la evidencia empírica apunta a que los supuestos ahorros que se obtienen mediante la concesión de los servicios públicos de agua potable, alcantarillado, saneamiento y 
gestión urbana de los residuos municipales son, si acaso, pequeños (Germà Bel y Warner 2008), los gobiernos locales han comenzado a buscar otras alternativas para minimizar el impacto financiero sobre sus comunidades. Bel y Mur han examinado la cooperación intermunicipal en España a través de comarcas (condados) y mancomunidades (asociaciones de municipios), encontrando que en el caso de la gestión urbana de los residuos municipales en la Región Autónoma de Aragón, la cooperación intermunicipal reduce costos en municipalidades con poblaciones más pequeñas (Germá Bel, Fageda, y Mur 2012, Germà Bel y Mur 2009).

La dimensión territorial, espacial/geográfica es una de las más importantes, si no es que tal vez la más relevante, dado que el surgimiento de un arreglo intermunicipal cooperativo es práctica y funcionalmente dependiente de la territorialidad (Cadaval y Caramés 2006). El territorio como variable funcional en el estudio de la cooperación entre agentes es también una tradición española de la investigación académica sobre intermunicipalidad. Por ejemplo, Nieto Garrido explica que la constitución española de 1978 contribuyó al desarrollo de este tipo de arreglos institucionales al reconocer el principio de la autonomía local (Hulst y Van Montfort 2007a: 169). En México, la autonomía local no forma parte de una reforma constitucional. Sin embargo, desde 1983 se reconoce la importancia de asignar a los municipios la provisión de los servicios públicos. Pero en la autonomía de los municipios también se conlleva la dimensión territorial.

Empíricamente, la determinación de la existencia de un arreglo intermunicipal de gobernanza de un servicio público resulta difícil de realizar, pero no imposible (ver por ejemplo, el reciente trabajo de Zachary Spicer (2014), si bien este autor solamente se enfoca en acuerdos firmados, o como diría Elinor Ostrom, reglas formales). En primer lugar, para determinar la posible intermunicipalización se puede acceder a evidencia de la expansión urbana y del estrechamiento de las divisiones geográficas y los bordes municipales. Esto se puede realizar con herramientas de sistemas de información geográfica y mapeo digital. En segundo lugar, es posible estudiar de manera etnográfica las relaciones entre los diferentes agentes encargados del suministro del servicio público. La etnografía institucional como método de estudio (McCauley 2014) ha tenido ya aplicaciones en el estudio de la gobernanza de los servicios públicos, en particular agua, si bien es un método relativamente nuevo (Mirosa 2012). En este artículo utilizo etnografía institucional tanto observando las relaciones de poder y los patrones de interacción entre los diferentes agentes responsables de la provisión del agua en las diferentes municipalidades, así como evaluando los textos relacionados con la posible emergencia de un arreglo intermunicipal. Esta última modalidad de etnografía institucional se 
puede denominar también en otras disciplinas como análisis de la retórica o análisis del discurso.

Un arreglo intermunicipal se reconoce no solamente cuando existe colindancia de fronteras (dimensión espacial/geográfica), sino también cuando existen relaciones cooperativas ampliamente visibles (dimensión colaborativa/institucional) en el manejo del servicio público. Es decir, no basta tener una zona metropolitana delimitada de jure sino de facto, es decir, uno reconoce el arreglo intermunicipal en el momento en el cual la cooperación entre los diferentes organismos operadores y los gobiernos municipales es evidente. Esto quiere decir que para que podamos reconocer un arreglo intermunicipal es necesario que se cumplan las condiciones de espacialidad interrelacionada, y de gobernanza cooperativa. Como indica Spicer, resulta particularmente interesante el estudio de la creación de acuerdos de cooperación intermunicipal en ciudades y condados que previamente no estaban conectados (Spicer 2014).

\section{¿CÓMO FUNCIONARÍA UN ARREGLO INTERMUNICIPAL DE GOBERNANZA DEL AGUA Y QUÉ EFECTOS POSITIVOS PODRÍA TENER?}

En general, los casos de cooperación intermunicipal en materia de agua han sido primordialmente aquellos en los cuales se establecen medidas y objetivos de eficiencia de los organismos operadores de agua (Armendáriz Torres 2010, IMCO 2014). El objetivo de un arreglo intermunicipal de gestión del recurso hídrico es reducir los costos de transacción y monitoreo (Warner y Hefetz 2011). En esta sección me enfoco primordialmente en el suministro del servicio público de saneamiento de efluentes residuales, si bien para términos del mismo también discuto brevemente aspectos de privatización del suministro de agua potable.

En esta sección del artículo postulo varias razones por las cuales un arreglo institucional de manejo intermunicipal del agua puede tener efectos positivos en la gobernanza del recurso hidrico. En primer lugar, la proximidad geográfica de los municipios podría ser de utilidad sobre todo para establecer interconexiones físicas (sistemas de tuberías, plantas de tratamiento e infraestructura hidráulica de distribución). Esta ventaja está directamente correlacionada tanto con la expansión urbana como con el incremento poblacional, y con la dimensión espacial y geográfica de la intermunicipalidad.

En segundo lugar, un arreglo institucional intermunicipal en materia de saneamiento permitiría establecer una relación organizacional consolidada y una serie de reglas formales sobre las competencias específicas de cada 
municipio, así como los mecanismos apropiados de asociación para el tratamiento y reuso de las aguas residuales en la zona metropolitana en cuestión (Pacheco-Vega 2007b). Para esto es importante que los organismos operadores de agua estén dispuestos a cooperar los unos con los otros, y que al menos exista un flujo de información substancial, continuo y en el cual exista credibilidad de un agente hacia el otro (Bressers y Lulofs 2010). En los casos en los cuales no existe monitoreo, credibilidad y confianza, así como sistemas de rendición de cuentas, el organismo operador de agua se debilita (Lutz Ley y Salazar Adams 2011, Pineda Pablos 2008), y con ello se debilita el arreglo cooperativo intermunicipal.

En tercer lugar, el establecimiento de mecanismos formales de cooperación en materia de tratamiento de aguas residuales pudiera sentar las bases para el desenmarañamiento de la compleja relación entre estado y municipio, así como el fortalecimiento de las relaciones cooperativas duraderas entre municipios, más allá de lo que se lograra mediante un arreglo cooperativo intermunicipal temporal.

En cuarto lugar, la construcción de capacidades técnicas en cada uno de los municipios que formara parte del sistema intermunicipal de tratamiento de aguas residuales podría también fortalecer sus propios sistemas de provisión y suministro de agua potable. La infraestructura de todo el sistema intermunicipal podría verse beneficiada de la cooperación particularmente en el aspecto financiero, ya que podría ser posible que las municipalidades que forman parte del arreglo institucional cooperativo pudieran compartir gastos de inversión en sistemas de tratamiento de aguas novedosos.

En quinto lugar, y más importante, la creación y fortalecimiento de arreglos de coordinación intermunicipal puede conllevar la consolidación real de una zona metropolitana, en lugar de ser solamente una zona metropolitana de nombre y no de facto. Esto es importante sobre todo desde la perspectiva de la gobernanza metropolitana (Lefevre 1998, Nelles y Durand 2012, Ramírez de la Cruz 2012, Taylor 2012).

\section{INTERMUNICIPALIDAD EN LA ZONA METROPOLITANA DE AGUASCALIENTES: ARREGLOS INSTITUCIONALES COMPLEJOS, EXPANSIÓN URBANA Y PRESIÓN HÍDRICA}

En esta sección realizo un recuento histórico del manejo del agua en dicha ciudad y en sus municipalidades conurbadas más allá de las discusiones sobre privatización. Asimismo, aplico el marco analítico de la intermunicipalidad a lo largo de mi descripción de la historia hidráulica de Aguascalientes, resaltando los momentos temporales en los cuales la 
dimensión territorial y la dimensión cooperativa emergente se hacen más relevantes. En particular, Aguascalientes es un caso de estudio sumamente relevante para el estudio de la intermunicipalidad dada la expansión urbana del mismo (Pacheco-Vega 2014b). Es interesante que una ciudad cuyo nombre recuerda precisamente el abundante suministro de agua (y que históricamente fue famosa por sus baños termales, de donde viene el nombre de Aguascalientes) carezca de un suministro adecuado del servicio de agua potable y alcantarillado.

Este fenómeno es doblemente sorprendente porque, desde una perspectiva de desarrollo urbano sustentable, el proceso evolutivo del agua en Aguascalientes ha mostrado un deterioro continuo debido a una serie de factores tanto biofísicos (cambio climático) como sociopolíticos (privatización y mercantilización del suministro) y urbanísticos (incremento de la población y transformación de la ruralidad del municipio hacia un desarrollo expandido urbano con una explosión en el crecimiento de construcciones de cotos privados y desarrollos habitacionales). El problema del agua en Aguascalientes es multifactorial, y cualquier análisis que pretende indicar solamente una causal es erróneo.

Si bien el enfoque central del artículo no es el proceso de la privatización del agua en México y específicamente en Aguascalientes (1993-1999), sí examino algunos de los elementos que han llevado a la transformación del proceso de provisión de agua en el municipio de Aguascalientes, que necesariamente incluyen la trayectoria de privatización (Amaya Ventura 2011, Caldera Ortega 2006, Pacheco-Vega 2014a, Pineda Pablos 1999, Torregrosa, Saavedra, y Kloster 2005). Es insoslayable entrar en una discusión sobre la privatización del suministro del agua y la transferencia de capacidades de operación de municipios a estados debido a que el fenómeno que ocurre en materia de suministro de agua puede también darse en aspectos de saneamiento y tratamiento de aguas residuales.

Una vez que la visión privatizadora entra en los paradigmas conceptuales de los políticos en el poder, es difícil poder combatirla. Este es precisamente el tipo de posicionamiento ideológico que ha creado conflictos en materia de privatización de agua en años recientes (Ahlers 2010, De Gouvello y Scott 2012). La filosofía neoliberal de implementar políticas públicas en las cuales el gobierno se aleja de la función de provisión de servicios en aras de mayor eficiencia, al ser provistos dichos servicios por una entidad privada es tentadora. Sin embargo, hay que examinar todas las posibles fuentes de ineficiencia en un sistema operador de agua potable y alcantarillado antes de transferir sus funciones operativas a un consorcio corporativo. 
En el artículo no se analiza en detalle la operación de los sistemas de agua potable y alcantarillado en las dos ciudades colindantes que forman parte de la zona metropolitana de Aguascalientes, Jesús María y San Francisco de los Romo, ya que no forma parte del análisis desarrollado. Sin embargo, por obvias razones (la cercanía geográfica y la continua migración temporal entre las tres municipalidades), tienen que incluirse en el estudio si bien no ser el punto central del análisis.

La zona metropolitana ofrece una serie de interesantes peculiaridades. En primer lugar, el suministro de agua en la ciudad de Aguascalientes está privatizado, mientras que en las ciudades colindantes de Jesús María y San Francisco de los Romo existen instancias públicas que ofrecen el servicio (con mayor o menor calidad, y con una gran variedad de niveles de servicio, medidos en número de horas conectados con suministro ininterrumpido). En segundo lugar, la localización espacial de ambas municipalidades y la expansión urbana acelerada que ha experimentado Aguascalientes prácticamente ha conurbado a Jesús María y a San Francisco de los Romo. En este proceso de conurbación de Aguascalientes, el comportamiento de los sistemas de distribución de agua ha sido fragmentado y cada municipalidad opera independientemente, en lugar de diseñar un sistema de manejo de aguas intermunicipal como lo han realizado las zonas metropolitanas de Pachuca (Hidalgo), Monterrey (Nuevo León), San Luis Potosí (San Luis Potosí) y Guadalajara (Jalisco).

El manejo de las aguas residuales en realidad es responsabilidad de cada uno de los municipios, de acuerdo con el artículo 115 constitucional y su reforma del año 1999. Muchas de las atribuciones de los municipios fueron establecidas en el año de 1983, sin embargo, agua potable, alcantarillado y saneamiento fueron integrados a las responsabilidades municipales en 1999 (López 2000) ${ }^{1}$. Sin embargo, en un caso extraño de traslape jurisdiccional y de concurrencia de competencias, el Instituto del Agua del Estado de Aguascalientes (INAGUA) se encuentra muy involucrado en el seguimiento de la operación de las plantas de tratamiento de aguas residuales en todo el estado.

Esta situación no es mucho de extrañar dado que hay otros estados en los cuales el seguimiento al agua residual ha dado frutos positivos, como el caso de Guanajuato, que si bien no tenía directamente el gobierno del agua residual en el estado, tuvo al menos en la década pasada, un liderazgo en materia de saneamiento debido al diseño explícito de políticas hídrica que fomentaran el diseño e implementación de plantas de tratamiento de aguas residuales (Pacheco-Vega 2009, 2012), particularmente durante el

I Agradezco el comentario acertado de un dictaminador anónimo de Revista de Gestión Pública sobre este punto en específico. 
periodo del Ing. Ricardo Sandoval Minero como Director Ejecutivo de la Comisión Estatal del Agua y Saneamiento de Guanajuato (CEAG).

\section{Mapa 1: El municipio de Jesús María, ubicado en contexto con el municipio de Aguascalientes}

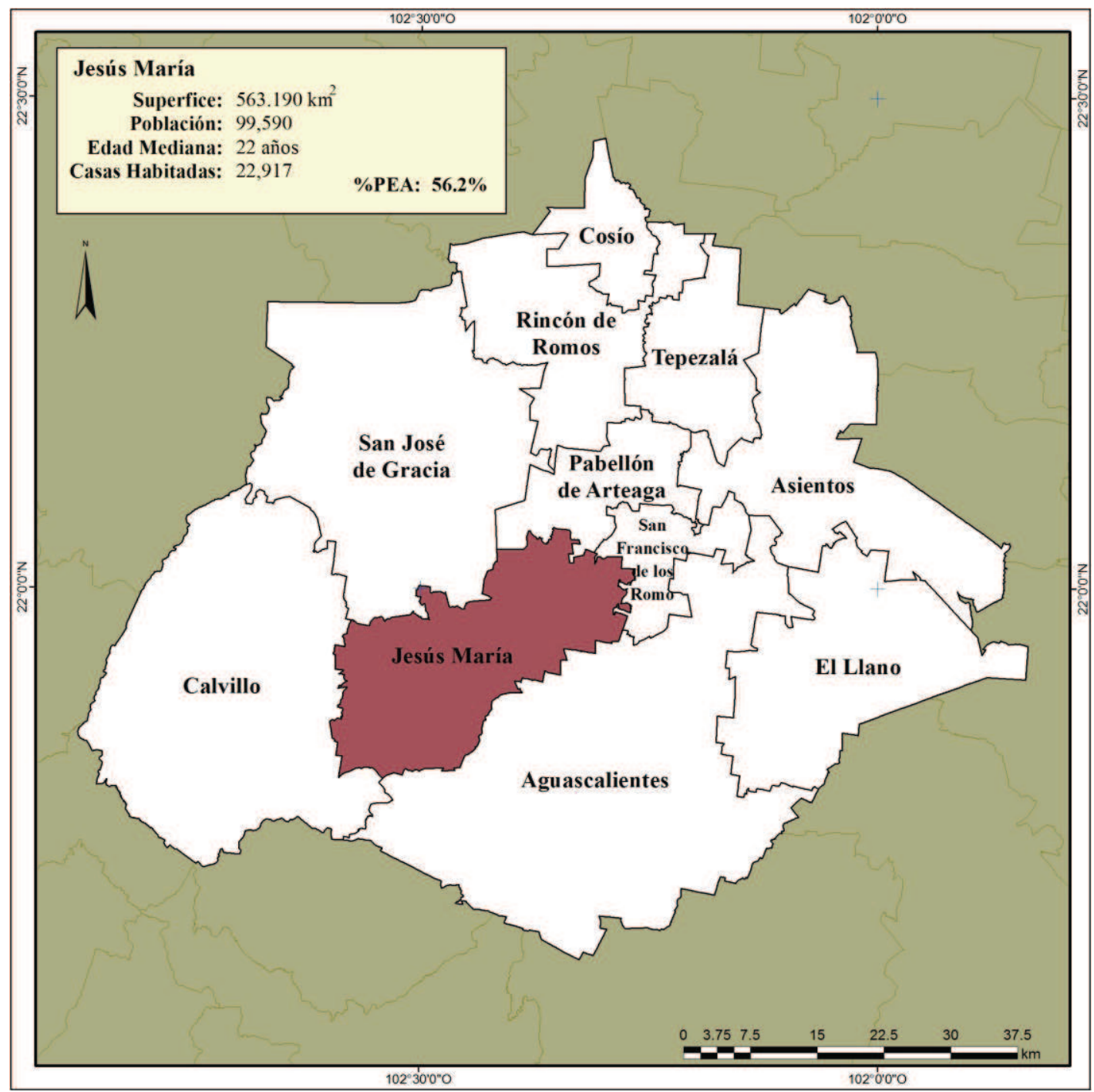

Fuente: http://www.aguascalientes.gob.mx/estado/municipios/mfps5.aspx [1408-2014].

El suministro de agua potable, alcantarillado y saneamiento en la zona metropolitana de Aguascalientes no deja de tener problemas por la excesiva construcción de nuevas viviendas en zonas colindantes entre los municipios ya conurbados. La municipalidad de Jesús María está ya, efectivamente, conurbada con la ciudad de Aguascalientes. Sin embargo, el suministro de agua en Jesús María se hace a través de la Comisión de Agua Potable, Alcantarillado y Saneamiento (CAPAS), todavía una entidad 
pública. No es de extrañarse que dicha comisión también ha enfrentado una serie de problemas también, posiblemente como resultado de la desalineación partidista al interior del Partido Revolucionario Institucional y a las presiones políticas en contra del recientemente relevado Presidente Municipal de Jesús María, Martín Chávez del Bosque.

Fuentes periodísticas indican que una auditoría reciente (2013) a la CAPAS arrojó irregularidades en el cobro del servicio de agua (La Jornada Aguascalientes 2012a). El Director de CAPAS fue cesado en febrero del 2013, después de dos años de quejas continuas por parte de los usuarios con respecto a la falta de suministro continuo de agua (El Clarinete 2013). Este problema en materia de suministro de agua se agudiza debido a la expansión urbana que ha convertido a Jesús María en el norte de Aguascalientes, con el consecuente crecimiento en construcción de cotos privados para residentes con un nivel económico medio a alto $^{2}$.

La posibilidad de que el municipio de Jesús María privatice su suministro de agua ha sido puesta sobre la mesa desde hace ya un par de ańos ${ }^{3}$. Resulta extraña esta actitud de estar interesados en la privatización, a pesar de que han sido documentados los efectos negativos que la concesión de agua ha tenido sobre la provisión adecuada del recurso hídrico en la municipalidad vecina de Aguascalientes (Amaya Ventura 2010, Caldera Ortega 2012, Pacheco-Vega 2013a, Pineda Pablos 2002).

Es importante señalar que el surgimiento de patrones irregulares de suministro de agua mediante pipas comerciales e industriales o tomas irregulares y clandestinas es resultado de una serie de problemáticas con relación al desabasto del vital líquido. Como en el caso del municipio de Aguascalientes, el suministro continuo del recurso hídrico ha sido difícil de alcanzar. En contraste con Aguascalientes, en Jesús María solamente se ha hablado de la posibilidad de otorgar una concesión a un operador privado, primordialmente debido a la falta de capacitación y a la ausencia de infraestructura adecuada tanto de perforación de pozos como de capacidad de bombeo y suministro a los hogares ${ }^{4}$.

2 Es curioso que la división entre el norte y el sur de Aguascalientes sigue, y que por ende, el sur de Jesús María se ha convertido gradualmente en el norte de Aguascalientes, lo cual se ha utilizado como herramienta mercadotécnica para poder vender terrenos y casas en cotos privados en el sur de Jesús María, a pesar del deficiente suministro del vital líquido. Para más información ver Lara (2012).

3 El ex Presidente Municipal y el Cabildo anteriores tenían la intención de poner sobre la mesa una propuesta de concesión del servicio (El Heraldo de Aguascalientes 20 I 2).

4 Esta problemática ya se había detectado desde el 20I2, y ha seguido hasta el 2014 (Noticiero El Circo 2013). 
Uno de los argumentos esgrimidos como razones suficientes para poder implementar un proceso de privatización es la concurrencia de dos factores: la incapacidad de la autoridad o el organismo operador de hacer cumplir las leyes y reglamentos en materia de suministro de agua potable en la escala local, y la falta de pago por parte de los usuarios ${ }^{5}$ del mismo. La localidad aparentemente ha tenido problemas con el mantenimiento de los pozos de abastecimiento desde el primero de enero del 2011.

Una grave realidad de la situación de Jesús María es que de los 120.000 habitantes de la municipalidad, solamente 26,000 se encuentran conectados al servicio de agua potable y alcantarillado. Pero aún peor resulta que la falta de cobro sea superior al $90 \%$ con 21.000 usuarios que no pagan el servicio (La Jornada 2012).

Si bien hay algunos indicios de una posible transferencia de políticas públicas (Benson y Jordan 2012, Dussauge-Laguna 2012, Pollitt 2002) entre municipios en la zona metropolitana de Aguascalientes, la hipótesis de que Jesús María aprendería de Aguascalientes resulta poco plausible, si bien la Alcaldesa de Aguascalientes, Lorena Martínez, quien gobernaba en el mismo periodo que Martín Chávez lo hacía en Jesús María, mencionó públicamente su apoyo a los procesos privatizadores del agua, cuando se le cuestionó su opinión en relación con los problemas de suministro y desabasto de agua que Jesús María afrontaba y que no han sido resueltos hasta la fecha (La Jornada Aguascalientes 2012b). A pesar de que la idea de una transferencia de políticas públicas entre municipios es interesante (entendida como el proceso de transmisión de los aprendizajes provenientes de ideas o acciones de política pública que pudiera aplicar un municipio al imitar a otro), el estudio de este posible proceso queda fuera de los límites y objeto del presente trabajo. Valga solamente resaltar que la posibilidad existe, sobre todo en municipios geográficamente colindantes (Drezner 2001, Rose 1991).

Desde la perspectiva de relaciones intermunicipales de coordinación, hay que tomar en cuenta también al municipio de San Francisco de los Romo. Este municipio alcanzó la categoría política como tal el 20 de febrero de 1904 , a pesar de que la propuesta por parte de una serie de cabilderos que propusieron al gobierno del ayuntamiento de Aguascalientes que la población de San Francisco de los Romo ya había alcanzado el nivel suficiente para ameritar ser un municipio, le fue denegado este nombramiento hasta 1977, momento en el cual se envió al Congreso del Estado una solicitud

5 En el caso específico de Jesús María, éste ha sido el argumento más socorrido (La Jornada 20I2). 
que también le fue rechazada a quienes la enviaron, finalmente teniendo éxito en 1992, momento en el cual el entonces Gobernador del Estado de Aguascalientes, ingeniero Miguel Ángel Barberena Vega, dio lugar a que se crearan dos nuevos municipios: El Llano y San Francisco de los Romo ${ }^{6}$.

De acuerdo con la información publicada sobre la pequeña ciudad, es considerada como un municipio al servicio de Aguascalientes, en el sentido en que hay dos parques industriales localizados en su territorio, el Parque Industrial del Valle de Aguascalientes (PIVA) y el Parque Industrial de San Francisco de los Romo, que albergan naves industriales para industria pesada $^{7}$. La ventaja comparativa y espacial de San Francisco de los Romo con respecto de Aguascalientes es que la vida es mucho más barata en esta zona geográfica.

Es importante hacer notar que dada la cercanía geográfica con Aguascalientes y el surgimiento del mismo municipio de una cabecera al norte, los mecanismos de gobernanza del recurso hídrico todavía se encuentran, en mucho, conectados con la forma en la que se maneja en agua en la municipalidad de Aguascalientes. Por ello es posible afirmar en base a la evidencia empírica presentada que existe una intermunicipalidad emergente en Aguascalientes y su zona metropolitana en relación con la gobernanza del agua.

6 Para una perspectiva histórica del tema, ver Ayuntamiento del Municipio de San Francisco de los Romo. http://sanfranciscodelosromo.gob.mx/portalweb/index.php/ municipio/historia [I4-08-20I4].

7 Para más información sobre los parques industriales, ver http://es.wikipedia.org/ wiki/San_Francisco_de_los_Romo_(municipio) [14-08-2014]. 


\section{Mapa 2: El municipio de San Francisco de los Romo, ubicado en contexto con el municipio de Aguascalientes.}

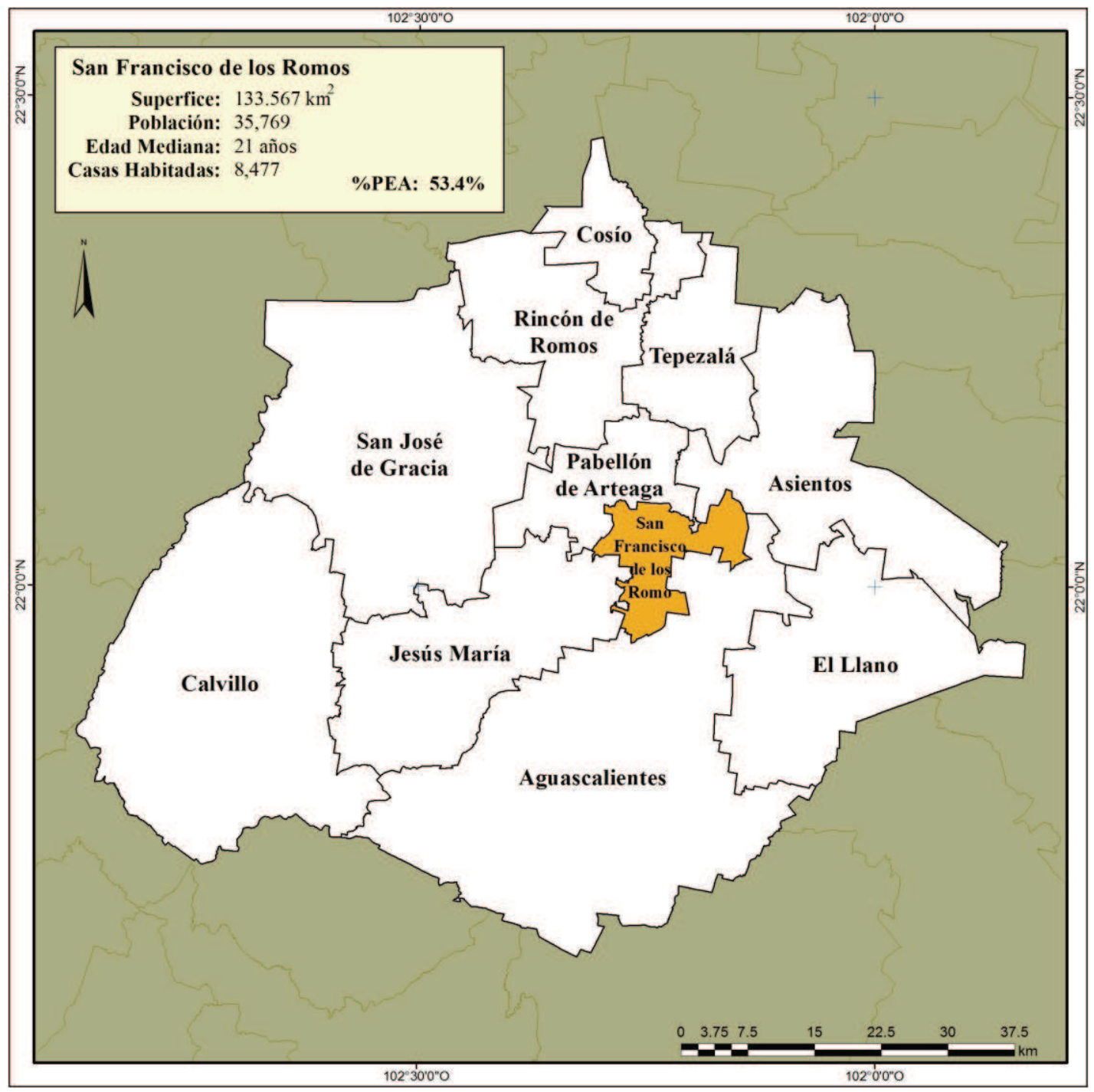

Fuente: http://www.aguascalientes.gob.mx/estado/municipios/mfps5.aspx [1408-2014].

Hay definitivamente plantas de tratamiento de aguas residuales localizadas en San Francisco de los Romo, pero determinar con exactitud la forma en la cual están gobernadas es complejo. La situación de manejo de aguas residuales en la zona metropolitana se hizo todavía más complicada en meses recientes cuando la licenciada Lorena Martínez dejó el cargo de Alcaldesa de la ciudad de Aguascalientes. Al dejar su cargo, la licenciada Martínez también dejó atrás una serie de proyectos importantes incluyendo la Línea Morada y las Líneas Verdes, ambos proyectos relacionados con un arreglo intermunicipal de manejo de agua. 


\section{CONCLUSIONES: ¿¿HACIA UNA INTERMUNICIPALIDAD COMPLEJA EN LA ZONA METROPOLITANA DE AGUASCALIENTES?}

A pesar de la popularidad de los análisis de arreglos institucionales en la literatura sobre gestión pública, poco es lo que se ha estudiado el arreglo institucional cooperativo intermunicipal, particularmente en el sector hídrico. En este artículo se analiza la posible emergencia de un arreglo institucional complejo de tipo intermunicipal donde el análisis sugiere que el cambio en las relaciones entre los diferentes municipios postula un modelo de provisión del líquido mediante mecanismos de coordinación entre diferentes municipalidades.

Como explico en secciones anteriores, uno de los arreglos de coordinación más interesantes en materia de provisión de agua y servicios de saneamiento es precisamente la intermunicipalidad (Rodríguez-Oreggia y Tuirán Gutiérrez 2006) ya que permite fortalecer la infraestructura de cada una de las municipalidades involucradas en el arreglo institucional. Las relaciones intermunicipales son importantes y poco estudiadas en materia de manejo de agua (Amaya Ventura 2011), si bien los análisis realizados hasta el momento se han enfocado en el manejo y gobierno del suministro del vital líquido, en lugar de examinar los aspectos que tienen que ver con el servicio de alcantarillado, tratamiento de efluentes y disposición final. Mi intención con este artículo fue contribuir al emergente estudio de la intermunicipalidad a través del estudio del caso del manejo de agua residual y saneamiento en la zona metropolitana de Aguascalientes. Mi análisis detalló la forma en la cual ha emergido un posible arreglo intermunicipal de gobernanza del agua, enfatizando dos dimensiones primordiales específicas al caso de Aguascalientes: el proceso de privatización del suministro de agua y el tratamiento de aguas residuales, así como la arquitectura institucional de la gobernanza del saneamiento en Aguascalientes.

En este artículo enfatizo el papel que un posible diseño de gobernanza del agua intermunicipal podría tener para el futuro del manejo del recurso hídrico en la zona metropolitana de Aguascalientes. Mi análisis demuestra que si bien la problemática de escasez del recurso hídrico está empezando a impactar substancialmente a los municipios colindantes de Aguascalientes (Jesús María y San Francisco de los Romo), todavía no existe evidencia de que haya una coordinación intermunicipal. Esto quiere decir que aún cuando existe un cierto grado de intermunicipalidad emergente por la dimensión espacial y geográfica, todavía no han surgido las relaciones, reglas formales e informales de colaboración entre los municipios. 
Esta circunstancia, donde la intermunicipalidad es emergente en la dimensión espacial pero no en la dimensión institucional, tiene implicaciones importantes para el estudio de la intermunicipalidad. Particularmente, este caso demuestra que para que la intermunicipalidad realmente funcione como arreglo institucional complejo y colaborativo, es necesario que ambas dimensiones estén coordinadas y vayan en paralelo. Es decir, una intermunicipalidad eficiente involucra tanto esquemas colaborativos robustos como un grado substancial de proximidad espacial.

La arquitectura institucional de manejo del recurso hídrico en el estado de Aguascalientes y su relación con el trabajo relacionado del municipio es sumamente compleja, lo cual ofrece un laboratorio excelente para tratar de desenmarañar la forma en la que se maneja el agua en el municipio. Por todas estas razones, Aguascalientes resulta un caso clásico de gobernanza compleja del recurso hídrico. Asimismo, en el trabajo exploro algunos elementos que han sido dejados de lado en análisis anteriores, como el aspecto de la estructura intermunicipal de la zona metropolitana de Aguascalientes. Cuando analizamos en forma aislada una municipalidad que ya se encuentra conurbada con otros dos municipios, es natural que se cree un vacío en nuestro conocimiento del mismo. Este trabajo pretende contribuir a aliviar esta deficiencia. 


\section{REFERENCIAS}

Ahlers, R. (2010). Fixing and Nixing: The Politics of Water Privatization. Review of Radical Political Economics, 42 (2), 213-230.

Alcalde Alderete, R. de M. (2003). La Politica Intergubernamental del Servicio de Agua Potable en San Luis Potosí, SLP 1989-2002. Tesis de maestría, El Colegio de San Luis, México.

Amaya Ventura, M. de L. (2010). Acción Pública, Instituciones y Efectividad de los Mecanismos de Cooperación en el Sistema de Gestión del Agua de Aguascalientes. Gestión y Política Pública, XIX (1), 37-77.

Amaya Ventura, M. de L. (2011). Aspectos Institucionales de la Gestión del Agua en Pachuca, Hidalgo. Revista Mexicana de Sociología, 3 (3), 509-537.

Armendáriz Torres, S. (2010). Organismos Operadores de Agua y la Nueva Gestión Hidrica: El Caso de Cuatro Municipios Conurbados de la Zona Metropolitana de la Ciudad de México. Tesis de maestría, Universidad Autónoma Metropolitana Azcapotzalco, México.

Bel, G. Fageda, X. y Mur, M. (2012). Does Cooperation Reduce Service Delivery Costs? Evidence from Residential Solid Waste Services. Journal of Public Administration Research and Theory, 24 (1), 85-107.

Bel, G. y Mur, M. (2009). Intermunicipal Cooperation, Privatization and Waste Management Costs: Evidence From Rural Municipalities. Waste Management, 29 (10), 2772-2778.

Bel, G. y Warner, M. (2008). Does Privatization of Solid Waste and Water Services Reduce Costs? A Review of Empirical Studies. Resources, Conservation and Recycling, 52 (12), 1337--1348.

. (2014). Inter-Municipal Cooperation and Costs: Expectations and Evidence. Public Administration, en línea primero, 1-16.

Benson, D. y Jordan, A. (2012). Policy Transfer Research: Still Evolving, Not Yet Through? Political Studies Review, 10, 333-338.

Bressers, H. y Lulofs, K. (Eds.). (2010). Governance and Complexity in Water Management. Creating Cooperation through Boundary Spanning Strategies. Northhampton: Edward Elgar.

Cadaval, M. y Caramés, L. (2006). Una Aproximación a los Modelos de Intermunicipalidad. Urban Public Economics Review, 6, 33-67. 
Caldera Ortega, A. R. (2006). Balance y Expectativas de la Gobernanza del Agua en Aguascalientes. Una Reflexión en torno a los Quince Años de Participación Privada en el Servicio de Agua Potable y Alcantarillado. En Olivares, R. y Sandoval-Minero, R. (Eds.), El Agua Potable en México: Historia Reciente, Actores, Procesos y Propuestas. Ciudad de México: Asociación Nacional de Empresas de Agua y Saneamiento, A.C.

Caldera Ortega, A. R. (2012). Las Ideas y el Proceso Político en las Estrategias para Hacer Frente a la Crisis del Agua: Dos Casos Mexicanos. Vetas: Revista Del Colegio de San Luis, Año II (4), 54-99.

Cohen, A. y McCarthy, J. (2014). Reviewing Rescaling: Strengthening the Case for Environmental Considerations. Progress in Human Geography, en línea primero, 1-23.

Comisión Nacional del Agua (CONAGUA). (2010). Los Consejos de Cuenca: Presente y Futuro. Ciudad de México: Gerencia de Consejos de Cuenca, Comisión Nacional del Agua.

De Gouvello, B. y Scott, C. A. (2012). Has Water Privatization Peaked? The Future of Public Water Governance. Water International, 37 (2), 87-90.

Digaetano, A. y Lawless, P. (1999). Urban Governance and Industrial Decline: Governing Structures and Policy Agendas in Birmingham and Sheffield, England, and Detroit, Michigan, 1980-1997. Urban Affairs Review, 34 (4), 546-577.

Dooley, L. M. (2002). Case Study Research and Theory Building. Advances in Developing Human Resources, 4 (3), 335-354.

Drezner, D. W. (2001). Globalization and Policy Convergence. International Studies Review, 3 (1), 53-78.

Dussauge-Laguna, M. I. (2012). On The Past and Future of Policy Transfer Research : Benson and Jordan Revisited. Political Studies Review, 10, 313-324.

El Clarinete. (2013). Cesan al Titular de CAPAS en Jesús María. 16 de febrero. Disponible en http://elclarinete.com.mx/cesan-al-titular-decapas-en-jesus-maria/ [14-08-2014].

El Heraldo de Aguascalientes. (2012). Pretenden Concesionar el Agua en Jesús María. 22 de octubre. Disponible en http://heraldo.mx/pretend- 
en-concesionar-el-agua-en-jesus-maria/ [14-08-2014].

Feiock, R. (2004). Politics, Institutions and Local Land-Use Regulation. Urban Studies, 41 (2), 363-375.

Fernández Ruiz, J. (2001). La Reforma Constitucional de Diciembre de 1999 al Artículo 115. Cuestiones Constitucionales, 4, 241-277.

Feiock, R. C. (2008). Metropolitan Governance and Institutional Collective Action. Urban Affairs Review, 44 (3), 356-377.

Flyvbjerg, B. (2011). Case Study. En Denzin, N. K. y Lincoln, Y. S. (Eds.), The Sage Handbook of Qualitative Research. Thousand Oaks, CA: SAGE.

Gilson, L. (2012). The Case-Study Approach. En Gilson, L. (Ed.), Health Policy and Systems Research: A Methodology Reader. Ginebra: Alliance for Health Policy and Systems Research and World Health Organization.

Griffin, C. B. (1999). Watershed Councils: an Emerging Form of Public Participation in Natural Resource Management. Journal of the American Water Resources Association, 35 (3), 505-518.

Hughes, S. (2012). Authority Structures and Service Reform in Multilevel Urban Governance: The Case of Wastewater Recycling in California and Australia. Urban Affairs Review, 49 (3), 381-407.

Hulst, R. y Van Montfort, A. (Eds.). (2007a). Inter-Municipal Cooperation. Dordrecht: Springer.

Hulst, R. y Van Montfort, A. (2007b). Inter-Municipal Cooperation: A Widespread Phenomenon. En Hulst, R. y van Montfort, A. (Eds.), Inter-Municipal Cooperation in Europe. Dordrecht: Springer.

Huppé, G. A., Creech, H. y Knoblauch, D. (2012). The Frontiers of Networked Governance. Winnipeg: International Institute for Sustainable Development.

Instituto Mexicano de la Competitividad (IMCO). (2014). Guía para la creación de Organismos Metropolitanos de Agua y Saneamiento en México. Ciudad de México: Instituto Mexicano de la Competitividad (IMCO) y Embajada Británica.

La Jornada. (2012). Edil Aguascalentense: Morisidad en Pagos de Agua Potable Abre Puerta a su Privatización. 16 de diciembre. Disponible en http://www.jornada.unam.mx/2012/12/16/estados/028n1est ([14- 
08-2014].

La Jornada Aguascalientes. (2012a). [Jesús María] Desabasto de Agua. 12 de octubre. Disponible en http://www.lja.mx/2012/10/jesus-mariadesabasto-de-agua/ [14-08-2014].

(2012b). Echando a Perder se Aprende: Lorena Martínez (A Propósito de las Concesiones de Agua). 23 de octubre. Disponible en http://www.lja.mx/2012/10/echando-a-perder-se-aprende-lorenamartinez-a-proposito-de-las-concesiones-de-agua/ [14-08-2014].

Lara, A. (2012). Pésimo Servicio de Agua en Jesús María. Disponible en http://www.televisaregional.com/aguascalientes/noticias/183349631. html [14-08-2014].

Lefevre, C. (1998). Metropolitan Government and Governance in Western Countries : A Critical Review. International Journal of Urban and Regional Research, 22 (1), 9-25.

López, M. P. (2000). La Reforma de 1999 al Artículo 115 Constitucional. El Nuevo Encuadramiento Fundamental del Municipio. Alegatos, 45, 287-306.

Lutz Ley, A. y Salazar Adams, A. (2011). Evolución y Perfiles de Eficiencia de los Organismos Operadores de Agua Potable en México. Estudios Demográficos y Urbanos, 26 (78), 563-599.

McCauley, J. (2014). Using Institutional Ethnography to Examine the Social Organization of Absence. Social Epistemology Review and Reply Collective, 3 (8), 22-27.

Mirosa, O. (2012). The Global Water Regime: Water's Transformation from Right to Commodity in South Africa and Bolivia. Tesis de doctorado, University of Wisconsin-Madison, Estados Unidos.

Montero, S. G., Castellón, E. S., Rivera, L. M. M., Ruvalcaba, S. G. y Llamas, J. J. (2006). Collaborative Governance for Sustainable Water Resources Management: The Experience of the Inter-Municipal Initiative for the Integrated Management of the Ayuquila River Basin, Mexico. Environment and Urbanization, 18 (2), 297-313.

Moynihan, D. P. (2009). The Network Governance of Crisis Response: Case Studies of Incident Command Systems. Journal of Public Administration Research and Theory, 19 (4), 895-915. 
Nelles, J. y Durand, F. (2012). Political Rescaling and Metropolitan Governance in Cross-Border Regions: Comparing the cross-Border Metropolitan Areas of Lille and Luxembourg. European Urban and Regional Studies, 21 (1), 104-122.

Noticiero El Circo. (2013). Se Incrementa la Cobertura del Servicio de Agua Potable en Zonas Marginales en Jesús María. 11 de noviembre. Disponible en http://www.noticieroelcirco.mx/?p=5601 [14-082014].

Ostrom, E. (2005). Understanding Institutional Diversity. Princeton: Princeton University Press.

Pacheco-Vega, R. (2007a). Construyendo Puentes entre la Política Ambiental y la Política de Tratamiento de Aguas en la Cuenca LermaChapala. Economía, Sociedad y Territorio, VI (24), 995-1024.

(2007b). Participación de la Comisión Nacional del Agua en el tratamiento de aguas residuales en la Cuenca Lerma-Chapala. Estadísticas federales y realidades estatales. Región y Sociedad, XIX (39), 55-76.

(2009). Arreglos Institucionales para el Saneamiento de Aguas Residuales en México. Un Caso de Estudio en la Cuenca LermaChapala. En Sandré Osorio, I., do Carmo, R. L., Vargas-Velázquez, S. y Guzmán, N. B. (Eds.), Gestión del Agua: Una Visión Comparativa entre México y Brasil. Jiutepec: Instituto Mexicano de Tecnología del Agua.

(2011). The Promises and Pitfalls of Lesson Drawing in Water Governance Research: What Can We Learn about Watershed Management from River Basin Councils and Organizations in Mexico. Ponencia presentada en el Annual Meeting of the Canadian Association of Geographers, 10 al 12 de marzo, Calgary, Alberta, Canada.

(2012). Arreglos Institucionales Dentro de la Cuenca LermaChapala: Una Visión desde la Política Ambiental. En Sánchez Rodríguez, M., Hernández López, J. de J., Durán Juárez, J. M. y Torres Rodríguez, A. (Eds.), Los Estudios del Agua en la Cuenca LermaChapala-Santiago. Agricultura, Industria y Ciudad. Pasado y Presente. Guadalajara y Zamora: Universidad de Guadalajara.

. (2013a). Geographies of Wastewater: A Comparative Analysis of Urban Sanitation Governance in the Mexican Municipalities of Aguascalientes (Aguascalientes) and Leon (Guanajuato). Ponencia 
presentada en el 2013 Meeting of the American Association of Geographers, 9 al 13 de abril Los Angeles, California, Estados Unidos.

. (2013b). Understanding the Dynamics of Institutional Erosion in Wastewater Governance processes through a Case Study of the Lerma-Chapala River Basin Council in Mexico. Ponencia presentada en la Biennial Conference of the International Association for the Study of the Commons (IASC), 3 al 7 de junio, Kitafuji, Japón.

. (2014a). La Gestión Urbana del Agua Residual en Aguascalientes: Una Mirada Neoinstitucionalista a la Privatización, el Saneamiento y el Reúso (2010-2013). Ponencia presentada en el III Congreso de la Red de Investigadores Sociales sobre el Agua (RISSA), 9 al 11 de abril, Salvatierra, Guanajuato, México.

(2014b). La Regionalización de la Gobernanza del Agua en Aguascalientes (1989-2014): Privatización, Reescalamiento e Intermunicipalidad. En Purón Cid, G. (Ed.), Coloquio del Proyecto Interdisciplinario de lo Regional. Aguascalientes, Aguascalientes: Centro de Investigación y Docencia Económicas, A.C. (CIDE).

Pacheco-Vega, R. y Basurto, F. (2008). Instituciones en el Saneamiento de Aguas Residuales: Reglas Formales e Informales en el Consejo de Cuenca Lerma-Chapala. Revista Mexicana de Sociología, 70 (1), 87109.

Pacheco-Vega, R. y Nemetz, P. N. (2001). Business-Not-As-Usual: Alternative Policy Instruments for Environmental Management. En IRE (Ed.), Proceedings of the 5th IRE Annual Workshop: Addressing the Knowledge Crisis in Water and Energy: Linking Local and Global Communities. Vancouver: Institute for Resources and Environment.

Pacheco-Vega, R. y Vega, O. (2008). Los Debates sobre la Gobernanza del Agua: Hacia una Agenda de Investigación en México. En Soares, D.,Vargas-Velázquez, S. y Nuño, R. (Eds.), La Gestión de Recursos Hidráulicos: Realidades y Perspectivas (Tomo I). Jiutepec, Morelos y Guadalajara, Jalisco: Instituto Mexicano de Tecnología del Agua y Universidad de Guadalajara.

Pasquini, L. y Shearing, C. (2014). Municipalities, Politics, and Climate Change: An Example of the Process of Institutionalizing an Environmental Agenda Within Local Government. The Journal of Environment \& Development, 23 (2), 271-296.

Pineda Pablos, N. (1999). Actores Sociales y Distribución de Costos y 
Beneficios en la Privatización del Agua Potable en Aguascalientes. Ciudades, 43 (1), 57-63.

(2002). La políticaUrbana de Agua Potable en México: Del Centralismo y los Subsidios a la Municipalización, la Autosuficiencia y la Privatización. Región y Sociedad, XIV (24), 41-69.

. (2008). Nacidos para Perder Dinero y Derrochar Agua. El Inadecuado Marco Institucional de los Organismos Operadores de Agua. En Soares, D., Vargas, S. y Nuño M. R. (Eds.), La Gestión de los Recursos Hidricos: Realidades y Perspectivas. Tomo 1. Jiutepec y Guadalajara: Secretaria de Medio Ambiente y Recursos Naturales, Instituto Mexicano de Tecnología del Agua, Universidad de Guadalajara.

Pollitt, C. (2002). Clarifying Convergence. Striking Similarities and Durable Differences in Public Management Reform. Public Management Review, 4 (1), 471-492.

Ramírez de la Cruz, E. E. (2012). Instituciones y Gobernanza Metropolitana: Una Primera Aproximación al Caso de México. Estudios Demográficos y Urbanos, 27 (2), 491-520.

Rodríguez-Oreggia, E. y Tuirán Gutiérrez, R. (2006). La Cooperación Intermunicipal en México. Barreras e Incentivos en la Probabilidad de Cooperar. Gestión y Politica Pública, XV (2), 393-409.

Rose, R. (1991). What is Lesson-Drawing? Journal of Public Policy, 11, 3-30.

Santos Zavala, J. (2004). Acción Pública Organizada: El Caso del Servicio de Agua Potable en la Zona Conurbada de San Luis Potosí. San Luis Potosí, México: El Colegio de San Luis, Miguel Ángel Porrúa, Universidad Autónoma Metropolitana.

Seawright, J. y Gerring, J. (2008). Case Selection Techniques in Case Study Research: A Menu of Qualitative and Quantitative Options. Political Research Quarterly, 61 (2), 294-308.

Spicer, Z. (2014). The Ties that Bind? Exploring the Dynamics of Intermunicipal Agreement Formation between Separated Cities and Counties. Canadian Public Policy, 40 (3), 245-258.

Taylor, J. J. (2012). The History of a Fluid Relationship: The Metropolitan Water District of Southern California and the San Diego County Water Authority to 1995. Tesis de maestría, California State University San 
Marcos, Estados Unidos.

Torregrosa, M. L., Saavedra, F. y Kloster, K. (2005). Posibilidades y Limitaciones de la Prestación de Servicios de Agua y Saneamiento: El Caso de Aguascalientes, México. Cuadernos Del CENDES, 22 (59), 89-109.

Vedung, E. (1998). Policy Instruments: Types and Theories. En M.L. Bemelmans-Videc, R. Rist, y E. Vedung (Eds.), Carrots, Sticks and Sermons: Policy Instruments and their Evaluation. New Jersey: Transaction Publishers.

Warner, M. E. y Bel, G. (2008). Competition or Monopoly? Comparing Privatization of Local Public Services in the Us and Spain. Public Administration, 86 (3), 723-735.

Warner, M. E. y Hefetz, A. (2011). Insourcing and Outsourcing: The Dynamics of Privatization among US Municipalities 2002-2007. Journal of the American Planning Association, 78 (3), 1-30.

Recibido: 05-09-2014

Aceptación de la versión final: 28-11-2014 